\title{
Radioembolization in Combination with Systemic Chemotherapy as First-line Therapy for Liver Metastases from Colorectal Cancer
}

\author{
Suzanne Kosmider, MBBS, Thean H. Tan, MBBS, Desmond Yip, MBBS, \\ Richard Dowling, MBBS, BMedSc, Meir Lichtenstein, MBBS, and \\ Peter Gibbs, MD
}

\begin{abstract}
Purpose: To report clinical experience with radioembolization (RE) plus systemic chemotherapy as a first-line treatment for liver metastases from colorectal cancer (CRC).

Materials and Methods: Clinical outcomes were evaluated retrospectively among 19 patients with unresectable liver metastases from CRC who had a good performance status and a low burden of extrahepatic disease (EHD) and were eligible for RE. Most (74\%) had disease confined to the liver. Concurrent treatment with 5-fluorourail/leucovorin $(\mathrm{n}=7)$ or 5-fluorourail/leucovorin/oxaliplatin (FOLFOX; $\mathrm{n}=12$ ) was started 3-4 days before single treatment with RE.

Results: Overall response rate according to the Response Evaluation Criteria in Solid Tumors was 84\% (two complete responses and 14 partial responses). Median progression-free survival (PFS) time was 10.4 months and median overall survival (OS) time was 29.4 months. For patients with disease confined to the liver, PFS improved (10.7 mo vs 3.6 mo; $P=.09)$, with significant prolongation of OS (median, $37.8 \mathrm{mo}$ vs $13.4 \mathrm{mo} ; P=.03$ ) compared with those who had EHD. Nine patients, including three long-term ( $>3$ y) survivors, remained alive after a median follow-up of 18.6 months. Serious treatment-related toxicities included febrile neutropenia with concurrent FOLFOX treatment, a perforated duodenal ulcer, and one death from hepatic toxicity.
\end{abstract}

Conclusions: The present findings confirm the effectiveness of RE plus systemic chemotherapy for metastatic CRC. Patients with liver-confined disease derived the greatest benefit, with median survival times beyond 36 months. Larger datasets from ongoing phase III trials are needed to further define the safety and efficacy of RE in the first-line setting.

\section{ABBREVIATIONS}

$\mathrm{CRC}=$ colorectal cancer, EHD $=$ extrahepatic disease, FOLFOX $=$ 5-fluorouracil/leucovorin/oxaliplatin, GDA = gastroduodenal artery, OS = overall survival, PFS = progression-free survival, RE = radioembolization, RECIST = Response Evaluation Criteria in Solid Tumors, RGA = right gastric artery

The liver is the most common site of metastatic disease in colorectal cancer (CRC). Radioembolization (RE) with yttrium-90 $\left({ }^{90} \mathrm{Y}\right)$ resin microspheres uses the well-characterized dual vasculature of the liver to selectively target tumors that are almost exclusively supplied by blood from the hepatic arterial branches while limiting the delivery to the normal liver parenchyma, which is supplied predominantly by the portal circulation (1). In most centers, RE is used as a salvage therapy for patients with liver-dominant metastatic disease (2-8). The clinical evidence from randomized controlled trials $(9,10)$ suggest that benefits may be greater if used earlier and in combination with systemic chemotherapy. To date, two randomized studies $(9,10)$ have reported significant benefits of combining RE with singleagent first-line chemotherapy, and more recently, encouraging outcomes were seen when RE was combined with 5-fluo-
From BioGrid Australia (S.K., P.G.), Level 6 North, Royal Melbourne Hospital, Grattan St., Parkville, Victoria 3050, Australia; Departments of Nuclear Medicine/Radiology (R.D., M.L.) and Oncology (P.G.), Royal Melbourne Hospital, Parkville, Victoria; and Department of Oncology (T.H.T., D.Y.), Canberra Hospital, Garran, Australian Capital Territory, Australia. Received October 13, 2009; final revision received January 31, 2011; accepted February 16, 2011. Address correspondence to S.K.; E-mail: suzanne. kosmider@mh.org.au.
D.Y. and P.G. are paid members of advisory board for Sirtex Medical (Sydney, Australia). None of the other authors have identified a conflict of interest.

(C) SIR, 2011

J Vasc Interv Radiol 2011; 22:780-786

DOI: $10.1016 /$ j.jvir.2011.02.023 
rouracil/leucovorin/oxaliplatin (FOLFOX) in a phase I dose-ranging study (11).

Here we report our early experience and long-term follow-up (to 78 mo after treatment) of patients with synchronous liver-dominant metastatic CRC who received RE combined with conventional intravenous chemotherapy as a first-line treatment.

\section{MATERIALS AND METHODS}

\section{Study Design and Inclusion Criteria}

The aim of our analysis was to assess retrospectively the clinical outcomes (overall response rate, progression-free survival [PFS], and overall survival [OS]) as well as the safety and tolerability of RE combined with systemic chemotherapy as first-line therapy among patients with synchronous liver metastases from CRC. We conducted a retrospective analysis of consecutive patients who were treated between January 2002 and October 2008 at one of two institutions. Institution review board approval was not required for this retrospective audit.

Patients with unresectable liver metastases from CRC, good performance status, and low burden of extrahepatic disease (EHD) at diagnosis (ie, limited pulmonary nodules or abdominal lymphadenopathy) were assessed as possible candidates for RE. Patients were encouraged to consider RE if a clinical trial option was not available, and aggressive therapy was indicated because of the bulk of disease or younger age of the patient. An unknown number of patients given the option of RE did not pursue this treatment option. All patients eligible for RE were required to have adequate renal function (ie, creatinine level $<1.5$ times normal value or creatinine clearance $>50 \mathrm{~mL} / \mathrm{min}$ ) and hemopoietic function (ie, leukocyte count $>1,500 / \mathrm{mm}^{3}$ and platelet count $>100,000 / \mathrm{mm}^{3}$ ) and sufficient liver function to tolerate RE (ie, absence of ascites or synthetic liver dysfunction, total bilirubin level $<1.75 \mathrm{mg} / \mathrm{dL}[<30 \mu \mathrm{mol} / \mathrm{L}$ ], and aspartate aminotransferase, alanine aminotransferase, and alkaline phosphatase levels each less than four times the upper limit of normal).

\section{Treatment Planning and RE}

A detailed account of the two-stage treatment protocol has been previously published (12). It comprised initial mapping angiography, branch coil embolization where indicated, and macroaggregated albumin scan. Typically, 2 weeks later, eligible patients underwent treatment of one or both sides of the liver by selective injection into the right and/or left hepatic arteries to avoid named or unnamed collateral vessels to the pancreaticoduodenal arcade or gastric arcades, or within the proper hepatic artery if no such collateral vessels were identified, as a single procedure with ${ }^{90} \mathrm{Y}$ resin microspheres (SIR-Spheres; Sirtex Medical, Sydney, Australia). The amount of microspheres administered was calculated based on an empiric or body surface area method as determined by the treatment center.

\section{Chemotherapy}

Patients were given oxaliplatin-based or 5-fluorouracil chemotherapy at clinician discretion and according to local protocols. RE was given on day 3 or 4 of cycle 1 . Chemotherapy was continued for a maximum of 6 months unless toxicity or disease progression necessitated treatment cessation.

\section{Assessments and Study Endpoints}

Patients were assessed by computed tomography (CT) of the chest, abdomen, and pelvis for tumor response at 8 weeks and every 2 months thereafter until disease progression was noted. The overall response rate was determined by Response Evaluation Criteria in Solid Tumors (RECIST) at the time of the best response recorded in the liver after RE. The durations of response and PFS were determined from the date of administration of RE until documented progression or, in those without disease progression, last review. OS was determined from the date of initiation of chemotherapy with RE to the documented date of death or last review for patients who were still alive.

Toxicities were graded according to National Cancer Institute Common Toxicity Criteria for Adverse Events, version 3. Adverse events were recorded from the time of the initiation of chemotherapy until the end of follow-up, documentation of disease progression, or voluntary withdrawal from the study.

The recently reported phase I dose-escalation trial of RE combined with increasing systemic doses of oxaliplatin (11) reported significant rates of grade $3 / 4$ neutropenia. Given this, it recommended an initial dose reduction of oxaliplatin to $65 \mathrm{mg} / \mathrm{m}^{2}$ (11). Many of the patients in the present study were treated before the study findings with full-dose oxaliplatin $\left(85 \mathrm{mg} / \mathrm{m}^{2}\right)$ were reported (11), so we specifically examined the rates of neutropenia in the first three treatment cycles for these patients.

\section{Statistical Analysis}

Kaplan-Meier survival analyses were applied, and comparisons were carried out by using the log-rank test. Data were analyzed using Stata software (version 10.0; Stata, College Station, Texas).

\section{RESULTS}

\section{Patient Characteristics and Treatment}

Nineteen patients received RE plus systemic chemotherapy and were included in the present analysis (Table 1). One patient had received 12 cycles of adjuvant FOLFOX chemotherapy for node-positive colon cancer with a relapsefree interval of 16 months before the development of liver metastases. All other patients had stage IV disease at initial presentation.

Median metastatic liver involvement, as determined by the treating clinician based on CT imaging, was $40 \%$ 
Table 1. Patient Characteristics and Concurrent

Chemotherapy at Baseline in RE Plus Systemic

Chemotherapy Study for Treating Liver Metastases from CRC

\begin{tabular}{lc}
\multicolumn{1}{c}{ Characteristic } & Value (N = 19) \\
Sex, $\mathrm{n}(\%)$ & $16(84)$ \\
Male & $3(16)$ \\
Female & \\
Median age (y) & 62 \\
Median & $44-75$ \\
Range & \\
ECOG performance status & $10(53)$ \\
0 & $9(47)$ \\
1 & $18(95)$ \\
Pts. with synchronous liver metastases & $5(26)$ \\
Pts. with EHD at diagnosis & \\
Liver involvement (\%) & 40 \\
Median & $25-65$ \\
Range & \\
ALP (IU/L) & 126 \\
Median & $97-535$ \\
Range & $3(16)$ \\
Pts. with CEA $>$ 200 ng/mL & \\
RE activity (GBq) & 1.96 \\
Median & $1.60-2.60$ \\
Range & \\
Concurrent chemotherapy & $7(37)$ \\
5-Fluorouracil & $12(63)$ \\
FOLFOX & $A L P$ \\
\hline
\end{tabular}

Note. - Values in parentheses are percentages. ALP = alkaline phosphatase; $\mathrm{CEA}=$ carcinoembryonic antigen; $\mathrm{ECOG}=$ Eastern Cooperative Oncology Group; EHD = extrahepatic disease; FOLFOX = 5-fluorouracil/leucovorin/oxaliplatin; RE = radioembolization.

(range, 25\%-65\%). The five patients with extrahepatic metastases had low-burden lung involvement with a limited number of pulmonary nodules (range, $1-8$ ) of small volume (range, $1-6 \mathrm{~mm}$ ).

Eleven of the 12 patients treated with concurrent FOLFOX chemotherapy were administered full-dose oxaliplatin $\left(85 \mathrm{mg} / \mathrm{m}^{2}\right)$ for the first three cycles of treatment. The remaining patient, who was treated after the publication of the phase I trial (11), received a lower dose of oxaliplatin $\left(65 \mathrm{mg} / \mathrm{m}^{2}\right)$ for the first three cycles.

A median activity of $1.96 \mathrm{GBq}$ (mean, $2.08 \mathrm{GBq}$; range, $1.60-2.60 \mathrm{GBq}$ ) of ${ }^{90} \mathrm{Y}$ resin microspheres was administered per patient as single whole-liver administration. Four of the 19 patients required a dose reduction of $20 \%$ as a result of pulmonary shunting of $10 \%-15 \%$ detected on a technetium-99m macroaggregated albumin scan before RE administration. None of the patients had greater than a $15 \%$ pulmonary shunt detected.

If the right and left hepatic arteries and gastroduodenal artery (GDA) arose as a trifurcation, embolization of the GDA was routinely performed to avoid potential
Table 2. Best Response Rates after Combined RE and

Systemic Chemotherapy ( $N=19$ ) in RE Plus Systemic

Chemotherapy Study for Treating Liver Metastases

from CRC

\begin{tabular}{ccc} 
& \multicolumn{2}{c}{ Response } \\
\cline { 2 - 3 } Outcome & Within Liver & Overall \\
CR & $2(11)$ & $2(11)$ \\
PR & $15(79)$ & $14(74)$ \\
SD & $1(5)$ & $1(5)$ \\
PD & $1(5)$ & $2(11)$ \\
\hline
\end{tabular}

Note. - Values in parentheses are percentages. $C R=$ complete response; $P D=$ progressive disease; $P R=$ partial response; $\mathrm{RE}=$ radioembolization; $\mathrm{SD}=$ stable disease.

reflux into the GDA. The right gastric artery (RGA) was embolized if positioning of the microcatheter beyond the RGA origin would have resulted in part of the right hepatic territory not being treated with RE. GDA embolization was required in four patients, and a fifth required embolization of the RGA and GDA. One additional patient had spasm of the left hepatic artery during RE, which required administration of a calcium channel blocker (verapamil).

\section{Tumor Response}

All patients were evaluated for disease response by RECIST. Two patients (11\%) with disease confined to the liver showed a complete response, $74 \%(n=14)$ showed a partial response, and $5 \%(n=1)$ showed stable disease (Table 2). One patient with a partial response in the liver had progression of pulmonary metastases and was considered to have progressive disease.

The median time to best response in the liver was 4.4 months (range, $0.9-50.3 \mathrm{mo}$ ). All patients who showed a partial response on CT imaging had a decrease in carcinoembryonic antigen level - which had been documented to be increased at baseline- 8 weeks after RE (median 35\% decrease; range, $19 \%-86 \%$ ).

\section{PFS}

Median PFS time was 10.4 months (Fig 1a). There was a trend for improved PFS in patients with liver-confined disease (10.7 mo vs $3.6 \mathrm{mo} ; P=.09$; Fig 1b). Median time to progression in the liver was 15.8 months.

In patients with disease progression $(n=12)$, the most common site of first progression was the lungs (eight of 12 patients; Table 3). When the liver was the first site of progression $(\mathrm{n}=7)$, it often occurred in conjunction with pulmonary progression $(\mathrm{n}=3$; Table 3$)$.

\section{Survival Outcomes}

The median OS for all patients was 29.4 months (Fig 2a). When patients were stratified by the presence or absence of EHD at diagnosis (Fig 2b), OS was superior in patients 


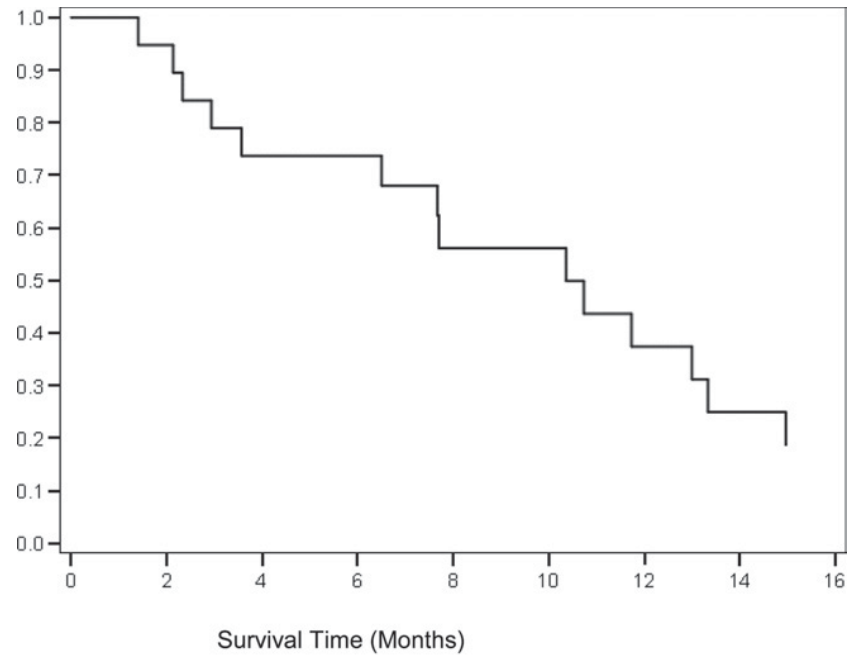

a.

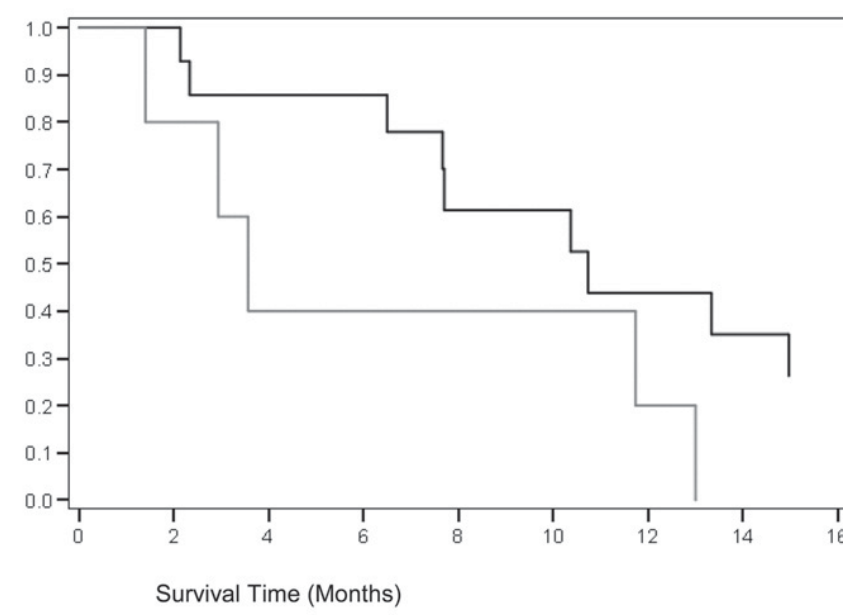

Extra-hepatic disease

No extra-hepatic disease

b.

Figure 1. PFS for all patients $(N=19)(a)$ and by presence $(n=$ $5)$ or absence ( $n=14$ ) of EHD (b).

Table 3. Site of First Metastatic Progression in Patients with Progressive Disease $(n=12)$

\section{Site}

\section{Patient Group}

EHD at diagnosis $(n=5)$

Hepatic disease only $(n=7)$

Note. $-E H D=$ extrahepatic disease.

with liver-only disease (median, 37.8 vs $13.4 \mathrm{mo} ; P=.03$ ). Nine of 19 patients remain alive after a median follow-up of 18.6 months (range, 3.2-78.5 mo). One patient has been disease-free per RECIST for more than 6 years, and two others remain in partial remission after more than 3 years of follow-up.

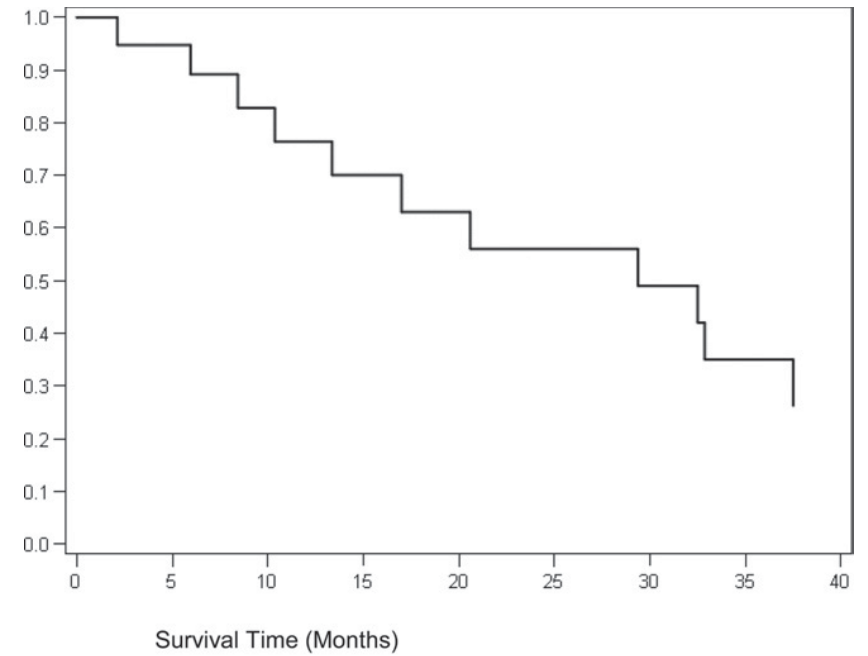

a.

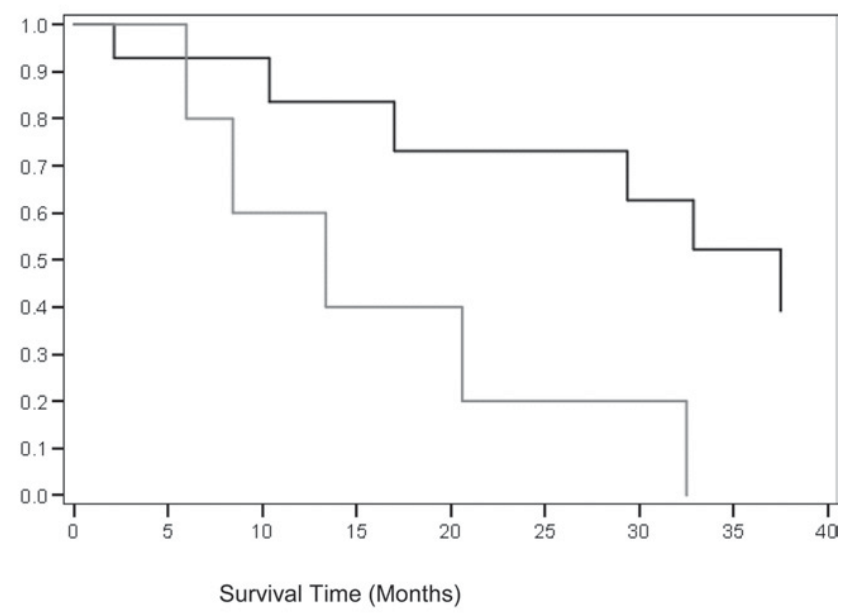

\section{Extra-hepatic disease}

No extra-hepatic disease

b.

Figure 2. OS for all patients $(\mathrm{N}=19)(\mathrm{a})$ and by presence $(\mathrm{n}=$ $5)$ or absence ( $n=14$ ) of EHD (b).

\section{Treatment Toxicities}

All adverse events of grade 2 or greater are listed in Table 4 at specified time intervals (1, 3, and $6 \mathrm{mo})$ after RE and chemotherapy. The majority of adverse events that occurred in the first month were abdominal pain, and seven patients had documented fever after RE as part of the well described post-RE syndrome (13). One patient who received FOLFOX chemotherapy with full-dose oxaliplatin $\left(85 \mathrm{mg} / \mathrm{m}^{2}\right)$ experienced an episode of febrile neutropenia within the first three cycles. No other patients had grade $3 / 4$ hematologic toxicity in this period.

The most serious adverse event was a treatment-related death from hepatic failure, presumed to represent radiation hepatitis, 2 months after treatment with RE and FOLFOX chemotherapy. At the time of RE, liver function was normal aside from an alkaline phosphatase level 1.5 times the upper 
Table 4. Adverse Events at 1, 3, and 6 Months after

Radioembolization and Chemotherapy ( $N=19)$

Grade 5

$\begin{array}{lcccc}\begin{array}{l}\text { Interval/Event } \\ \text { 0-4 Weeks }\end{array} & \text { Grade } 2 \text { Grade 3 Grade 4 } & \text { (Death) } \\ \text { Febrile neutropenia } & - & 1 & - & - \\ \text { Abdominal pain } & 4 & 3 & - & - \\ \text { Fatigue } & 6 & 4 & - & - \\ \text { 4-12 Weeks } & & & & \\ \text { Bilirubin } & 4 & 1 & - & - \\ \text { AST } & 4 & 1 & - & - \\ \text { ALP } & 4 & 1 & - & - \\ \text { Gastritis } & 2 & 4 & - & - \\ \text { Gastric ulceration } & - & - & 1 & - \\ \text { Liver dysfunction } & 4 & - & - & 1 \\ \text { Anorexia } & 6 & 3 & 1 & - \\ \text { Ascites } & 4 & 1 & - & - \\ \text { 12-24 Weeks } & & & & - \\ \text { Neuropathy } & 2 & 1 & - & - \\ \text { Hand-foot syndrome } & - & 1 & - & -\end{array}$

Note.-Adverse events are classified per National Cancer Institute Common Toxicity Criteria for Adverse Events, version 3. ALP = alkaline phosphatase; AST = aspartate aminotransferase.

limit of normal. The patient was older than 65 years of age with extensive liver metastasis $(60 \%$ involvement), and, because of pulmonary shunting, had a reduced dose of microspheres (calculated according to body surface area method at $2.0 \mathrm{GBq}$ ) infused via the proper hepatic artery to the whole liver.

Four other patients experienced transient increases in liver function test results and self-limiting low-volume ascites within 60 days after treatment, unrelated to progressive disease, which normalized to baseline levels within 2 weeks.

All patients received prophylaxis before $\mathrm{RE}$ with $\mathrm{H} 2$ antagonists or proton pump inhibitors, which continued for the duration of chemotherapy; however, six patients reported symptoms suggestive of gastroduodenitis within 6-12 weeks. Three of the six patients underwent GDA embolization before RE. Gastroduodenitis was confirmed by endoscopy in three cases, with symptoms lasting less than 3 weeks. One patient with severe symptoms at 12 weeks after RE was diagnosed with a perforated duodenal ulcer. Yttrium-90 resin microspheres were identified in a biopsy specimen taken from the duodenal wall. This patient had not undergone embolization of the GDA or RGA before RE.

Longer-term toxicities at 6 months were mainly related to chemotherapy (eg, oxaliplatin-induced neuropathy, 5-fluorouracil-related hand-foot syndrome.) One patient had splenomegaly on CT scan at 6 months after RE, which was not present on pretreatment imaging, with associated grade 1 thrombocytopenia $\left(<150-75,000 / \mathrm{mm}^{3}\right)$.

\section{DISCUSSION}

The present analysis confirms the activity of RE reported in the two previous randomized controlled trials $(9,10)$, both of which found higher response rates and improved PFS with RE combined with fluoropyrimidine chemotherapy compared with chemotherapy alone.

The optimal time to use RE with ${ }^{90} \mathrm{Y}$ resin microspheres in the treatment of CRC liver metastases remains uncertain. A recent phase I dose-escalation trial (11) explored the combination of RE with systemic FOLFOX chemotherapy in patients with chemotherapy-naive metastatic CRC. Toxicity was acceptable, partial responses were seen in 18 of 20 patients (90\%), median PFS time was 9.3 months, and median time to progression in the liver was 12.3 months. The results compare well with those of the present series (response rate of $84 \%$, median PFS of 10.4 mo, and median time to liver progression of $15.8 \mathrm{mo}$ ). Although it is not possible to compare the results of studies in patients who have received multiple previous lines of therapy versus those of studies with the added benefit achieved by the addition of RE in the first-line setting, some observations can be made. The largest published series of pretreated patients, by Kennedy et al (2), reported a response rate of $35 \%$. This is consistent with our own previously published experience (8) in which the overall response rate was $33 \%$, with a median PFS of 5.3 months, when RE was part of a second- or third-line treatment $(\mathrm{n}=$ 30 ); a response rate of $21 \%$ and median PFS time of 3.9 months were found when RE was used as third-line therapy $(\mathrm{n}=14)(8)$. The additional benefit of RE in terms of response rate compared with chemotherapy alone is approximately $30 \%$ in the first-line setting. This is similar to the response seen in pretreated patents. Ultimately, the most important issue will be to determine the benefit, in terms of impact on PFS and OS, of first-line treatment versus a later treatment setting.

Response to RE is typically slow $(2,14)$. In the present series, the median time to best response as assessed by CT was 4.4 months (range, 0.9-50.3 mo), and others have reported a median time as long as 12 months (15). In the patients in the present series, carcinoembryonic antigen levels decreased by a median of $35 \%$ within 6 weeks of RE and predicted response in all cases. It is worth noting that the use of RECIST criteria to assess disease response to RE may underestimate the number of patients who show a benefit from treatment. Residual lesions are often seen on $\mathrm{CT}$ in patients with a good response based on other criteria such as positron emission tomographic imaging or carcinoembryonic antigen measurement. In the study by Kennedy et al (2), 35\% of patients showed a response based on CT criteria, but a much higher percentage of patients showed a benefit as assessed by a change in positron emission tomographic imaging $(90 \%)$ or a decrease in carcinoembryonic antigen level (70\%) (2). In the present series, two patients with persistent abnormalities on CT have not shown disease progression more than 3 years after treatment. 
Particularly encouraging in the present series were the outcomes in patients with liver-confined metastatic disease, with a median PFS time of 10.7 months and a median OS time extending beyond 36 months. Even in the present small series, a significant difference in OS was observed between patients with liver-only disease and those with EHD (37.8 mo vs 13.4 mo; $P=.03)$, as seen in other clinical studies $(8,14)$.

$\mathrm{RE}$ is generally well tolerated, with a low incidence of RE-induced liver disease (13,16-18). The incidence of REinduced liver disease increases with increasing age, wholeliver treatment, and increased baseline bilirubin levels $(13,18)$. It can produce significant morbidity but is rarely life-threatening (16). Transient increases in liver function test findings and self-limiting low-volume ascites within 60 days after treatment are not life-limiting. The median OS for the four patients in the present study who experienced this complication was 25 months.

A treatment-related death from presumed radiation hepatitis occurred 2 months after RE and FOLFOX chemotherapy. After examining the specific details of this case, we believe this outcome could not have been predicted. There was no evidence of radiation pneumonitis in the series. This complication has not been reported since the implementation of routine screening to exclude patients with a significant liver-to-lung shunt (19).

The most common serious complication of RE results from inadvertent distribution of ${ }^{90} \mathrm{Y}$ microspheres into the lining of the gut, presumably via unidentified collateral vessels, resulting in gastritis or duodenitis. Six patients $(32 \%)$ in the present series developed symptoms suggestive of gastroduodenitis despite all receiving prophylaxis; only three of the cases were confirmed by endoscopy.

There was an unexpectedly high rate of complications in the present study. A detailed literature review of complications after RE (13) reported an incidence of gastric ulceration of less than $5 \%$ if proper percutaneous techniques are used. In this review (13), the highest reported rate of gastrointestinal ulceration was $29 \%$ (20). The present results may be an anomaly in view of the small sample size (and wide CIs), but may also reflect the learning curve of the interventionists. Unfortunately, this complication can occur even with routine embolization of the GDA and RGA in all patients in the presence of variant anatomy or incomplete embolization $(17,21)$.

We recognize that our analysis, although it provides an early indicator of the potential of RE in the first-line setting, cannot be used to draw definitive conclusions on the safety and efficacy of this treatment. Both the size of the analysis and the absence of a control arm are clear limitations of the study. The results of two ongoing international randomized controlled studies comparing chemotherapy with and without RE with ${ }^{90} \mathrm{Y}$ resin microspheres for the de novo treatment of patients with liver metastases from CRC will provide a much clearer indication of the risks and benefits in the first-line setting. In these trials, patients are stratified by presence of extrahepatic metastases and degree of liver involvement. Only a minority of patients with low-volume EHD (fewer than five lung nodules $<1 \mathrm{~cm}$ in diameter, and/or abdominal lymph nodes all $<$
$2 \mathrm{~cm}$ in diameter) will be included. Based on our experience, we would expect the analysis of patients with and without EHD to be of particular interest.

In conclusion, the present early series provides some valuable insights into the potential of RE when used in combination with chemotherapy for the initial treatment of patients who present with liver-dominant metastases from CRC. Patients with liver-only disease derived the greatest benefit, with median survival times beyond 36 months. However, results from larger datasets, and particularly from the ongoing phase III trials, are needed before this treatment can be recommended more widely as a first-line treatment for CRC liver metastases.

\section{REFERENCES}

1. Archer SG, Gray BN. Vascularization of small liver metastases. Br J Surg 1989; 76:545-548.

2. Kennedy AS, Coldwell D, Nutting C, et al. Resin 90Y-microsphere brachytherapy for unresectable colorectal liver metastases: modern USA experience. Int J Radiat Oncol Biol Phys 2006; 65:412-425.

3. Murthy $\mathrm{R}$, Xiong $\mathrm{H}$, Nunez $\mathrm{R}$, et al. Yttrium 90 resin microspheres for the treatment of unresectable colorectal hepatic metastases after failure of multiple chemotherapy regimens: preliminary results. J Vasc Interv Radiol 2005: 16:937-945

4. Popperl G, Helmberger T, Munzing W, Schmid R, Jacobs TF, Tatsch K. Selective internal radiation therapy with SIR-Spheres in patients with nonresectable liver tumors. Cancer Biother Radiopharm 2005; 20:200-208.

5. Wong CY, Qing F, Savin M, et al. Reduction of metastatic load to liver after intraarterial hepatic yttrium-90 radioembolization as evaluated by [18F]fluorodeoxyglucose positron emission tomographic imaging. J Vasc Interv Radiol 2005; 16:1101-1106.

6. Szyszko T, Al-Nahhas A, Tait P, et al. Management and prevention of adverse effects related to treatment of liver tumours with 90Y microspheres. Nucl Med Commun 2007; 28:21-24.

7. Jakobs TF, Hoffmann RT, Poepperl G, et al. Mid-term results in otherwise treatment refractory primary or secondary liver confined tumours treated with selective internal radiation therapy (SIRT) using (90)yttrium resin-microspheres. Eur Radiol 2007; 17:1320-1330.

8. Lim L, Gibbs P, Yip D, et al. A prospective evaluation of treatment with selective internal radiation therapy (SIR-spheres) in patients with unresectable liver metastases from colorectal cancer previously treated with 5-FU based chemotherapy. BMC Cancer 2005; 5:132-138.

9. Van Hazel G, Blackwell A, Anderson J, et al. Randomised phase 2 trial of SIR-Spheres plus fluorouracil/leucovorin chemotherapy versus fluorouracil/leucovorin chemotherapy alone in advanced colorectal cancer. J Surg Oncol 2004; 88:78-85.

10. Gray B, Van Hazel G, Hope M, et al. Randomised trial of SIR-Spheres plus chemotherapy vs. chemotherapy alone for treating patients with liver metastases from primary large bowel cancer. Ann Oncol 2001; 12:1711-1720.

11. Sharma RA, Van Hazel GA, Morgan B, et al. Radioembolization of liver metastases from colorectal cancer using yttrium-90 microspheres with concomitant systemic oxaliplatin, fluorouracil, and leucovorin chemotherapy. J Clin Oncol 2007; 25:1099-1106.

12. Lewandowski RJ, Sato KT, Atassi B, et al. Radioembolization with $90 \mathrm{Y}$ microspheres: angiographic and technical considerations. Cardiovasc Intervent Radiol 2007; 30:571-592.

13. Riaz A, Lewandowski RJ, Kulik LM, et al. Complications following radioembolization with yttrium-90 microspheres: a comprehensive literature review. J Vasc Interv Radiol 2009; 20:1121-1130.

14. Stubbs RS, O'Brien I, Correia MM. Selective internal radiation therapy with $90 \mathrm{Y}$ microspheres for colorectal liver metastases: single-centre experience with 100 patients. ANZ J Surg 2006; 76:696-703.

15. Boppudi S, Wickremesekera SK, Nowitz M, Stubbs R. Evaluation of the role of CT in the assessment of response to selective internal radiation therapy in patients with colorectal liver metastases. Australas Radiol 2006; 50:570-577

16. Kennedy AS, McNeillie P, Dezarn WA, et al. Treatment parameters and outcome in 680 treatments of internal radiation with resin 90Y-micro- 
spheres for unresectable hepatic tumors. Int J Radiat Oncol Biol Phys 2009; 74:1494-1500.

17. Murthy R, Brown DB, Salem R, et al. Gastrointestinal complications associated with hepatic arterial yttrium-90 microsphere therapy. J Vasc Interv Radiol 2007; 18:553-561.

18. Sangro B, Gil-Alzugaray B, Rodriguez J, et al. Liver disease induced by radioembolization of liver tumors: description and possible risk factors. Cancer 2008; 112:1538-1546.

19. Leung TW, Lau WY, Ho SK, et al. Radiation pneumonitis after selective internal radiation treatment with intraarterial 90yttrium-microspheres for inoperable hepatic tumors. Int J Radiat Oncol Biol Phys 1995: 33:919-924.

20. Neff R, Abdel-Misih R, Khatri J, et al. The toxicity of liver directed yttrium-90 microspheres in primary and metastatic liver tumors. Cancer Invest 2008; 26:173-177.

21. Lewandowski RJ, Thurston KG, Goin JE, et al. 90Y microsphere (TheraSphere) treatment for unresectable colorectal cancer metastases of the liver: response to treatment at targeted doses of 135-150 Gy as measured by [18F]fluorodeoxyglucose positron emission tomography and computed tomographic imaging. J Vasc Interv Radiol 2005; 16:1641-1651. 